\title{
Constraint-Variable Analysis of Time Coupling Constraints in Unit Commitment
}

\author{
Shuai $\mathrm{CAO}^{1}$, Zhenglin YANG ${ }^{2}$, Haihua $\mathrm{CHENG}^{3}$, \\ Yaxian ZHENG $^{4}$, Jian GENG ${ }^{5}$ \\ China Electric Power Research Institute, Nanjing 210003, China \\ ${ }^{1}$ sunever@qq.com, ${ }^{2}$ yangzhenglin@epri.sgcc.com.cn, ${ }^{3}$ chenghaihua@epri.sgcc.com.cn, ${ }^{4}$ zhengya \\ xian@epri.sgcc.com.cn, ${ }^{5}$ gengjian@epri.sgcc.com.cn
}

Keywords: constraint-variable correlation, time coupling constraints, unit commitment.

\begin{abstract}
Constraint-variable correlation is the most important coupling correlation in unit commitment problem. Reasonable arrangements for the correlation will simplify the model complexity, and reduce the calculation time. And minimum on/off time constraint is one of the most complicated time coupling constraints. The authors built 4 kinds of models of minimum on/off time constraints, and compared the different performances in constraint-variable correlation. It is clarified that the selected model were in better constraint-variable correlation mathematically. Finally, IEEE118 case was used to validate the analysis above.
\end{abstract}

\section{Introduction}

Unit commitment (UC) is to work out the problem of the on/off states and powers of specified units to satisfy the optimal objective on different time scales. Actually it is a problem of mixed integer programming (MIP). The coefficient matrix dimension grows up exponentially with the scale. It is significant for modeling the time coupling constraints well in constraint-variable correlation (CVC), especially the minimum on/off time constraints. The branch and cut (B\&C) is one of the most popular methods in business software, and the excellent model will improve the efficiency of B\&C [1]. There are some papers modeling and analyzing the minimum on/off time constraints. The 3-binary states was modeled in [2], furthermore [3] imposed the modified 3-binary model, coupling the startup state and running state. The 1-binary model formulated in [4] was proved to have better quality than [3] in saving the programming time and objective costs. But another case in [5] demonstrated the model of [3] was superior to [4] in node numbers. None papers above compared the models in CVC. It needs some convincing optimal criteria.

\section{Constraint-variable Correlation Analysis}

The optimization objective of UC problem is usually the total cost of system and the constraint conditions includes system power balance, reserve constraints, generation limits and ramping constraints, minimum on and off time constraints, etc. [6].

The UC model of MIP can be expressed by the symbolic form below.

$$
\min \left\{\mathbf{c}^{\mathrm{T}} \mathbf{x} \mid \mathbf{A x} \geq \mathbf{b}, \mathbf{x} \in \mathrm{N}^{\mathrm{n}}+\mathrm{R}_{*}^{\mathrm{n}}\right\}
$$

where $\mathbf{x}$ is a $n+r$ dimension column vector, $\mathbf{A}$ is a $k \times(n+r)$ dimension matrix,

The project is supported by the Science and Technology of SGCC (DZN17201500036). 
where $k$ is the order of maximal linear independent group.

It is expected that the feasible domain is as accurate as possible, and the CVC of the model is as concise as possible. CVC mainly analyzes the scale of constraints and variables, as well as the single incidence matrix. With row (column) single incidence, coefficient matrix A can be in reduced order through the elementary transformation [6], as shown in Fig.1. Ideal coefficient matrix A should be the diagonal or the Jordan canonical form. However, there are a large number of practical constraints, so the row rank is huge. Magnification and shrinking of the inequalities will effectively reduce the row rank, namely the number of linear independent valid inequalities.

$$
\mathbf{A}=\left(\begin{array}{ccccc}
\mathbf{A}_{1} & \mathbf{B}_{1} & & & \\
\mathbf{A}_{2} & & \mathbf{B}_{2} & & \\
\cdots & & & \ddots & \\
\mathbf{A}_{\mathbf{n}} & & & & \mathbf{B}_{\mathbf{n}}
\end{array}\right) \longrightarrow \begin{gathered}
\text { order } \sum_{\mathrm{i}=1}^{\mathrm{n}} \mathrm{m}_{\mathrm{i}} \text { reduced to } \mathrm{m}_{0}+\mathrm{n} \\
\mathbf{A}_{\mathrm{i}} \in \mathrm{R}^{\mathrm{n}_{\mathrm{i}} \times \mathrm{m}_{0}}, \mathbf{B}_{\mathrm{i}} \in \mathrm{R}^{\mathrm{n}_{\mathrm{i}} \times \mathrm{m}_{\mathrm{i}}}, \quad \text { column full rank }
\end{gathered}
$$

Figure 1. The single incidence matrix and deflation processing

In the condition of different constrained variables, the paper established 4 kinds of models, respectively named A, B, C, and D. All models can be formulated as

$$
\begin{aligned}
& \mathrm{A}\left\{\begin{array} { l } 
{ \sum _ { \tau = t + 1 } ^ { \operatorname { m i n } \{ t + M U T - 1 , T \} } u _ { i \tau } - ( u _ { i t } - u _ { i ( t - 1 ) } ) \geq 0 } \\
{ \sum _ { \tau = t + 1 } ^ { \operatorname { m i n } \{ t + M D T - 1 , T \} } 1 - u _ { i \tau } - ( u _ { i ( t - 1 ) } - u _ { i t } ) \geq 0 }
\end{array} \quad \mathrm { B } \left\{\begin{array}{l}
\sum_{\tau=t+1}^{\min \{t+M U T-1, T\}}\left(u_{i \tau}-y_{i t}\right) \geq 0 \\
\sum_{\tau=t+1}^{\min \{t+M D T-1, T\}}\left(1-u_{i \tau}-z_{i t}\right) \geq 0
\end{array}\right.\right. \\
& \mathrm{C}\left\{\begin{array}{l}
\sum_{\tau=t+1}^{\min \{t+M D T-1, T\}} y_{i \tau} \leq 1-u_{i t} \\
\sum_{\tau=t+1}^{\min \{t+M D T-1, T\}} y_{i \tau} \leq 1-z_{i t} \\
\sum_{\tau=t+1}^{\min \{t+M U T-1, T\}} z_{i \tau} \leq u_{i t}
\end{array} z_{i t} \leq 1-y_{i t}\right.
\end{aligned}
$$

Where $\mathrm{u}$ is the unit running state, $\mathrm{y}$ is unit start-up state, $\mathrm{z}$ is the shut-down state.

Model A means that after the start-up (or shut-down), units must remain the on (or off) states for at least the minimum period. The unit states of some time periods will be limited strictly by the initial time. Where $M U T$ represents the minimum on time, and $M D T$ represents the minimum off time. According to Eq.2, the threshold variable of model B is bigger than the A. Considering the inequality direction, constrained variables are in enhanced limits, and the feasible region of the relaxed problem becomes smaller. In theory, the effect of the model B is better than A. However for the introduction of independent integer variables, the model B will show the better performance after a certain time period.

$$
\left\{\begin{array}{l}
u_{i t}-u_{i(t-1)}=y_{i t}-z_{i t} \\
y_{i t}+z_{i t} \leq 1
\end{array}\right.
$$

Model $\mathrm{C}$ has the different modeling routine, focused on the difference of state variables, rather than state variables. Hence the amount of effective limited constraint is larger than model A or B, but limited variables in each constraint are smaller in quantity. To model D, although the amount of effective limited constraint is less, and also limited variables are better in each constraint, the coupling degree is the highest among all models.

\section{Numerical Results}

The proposed formulations have been applied to solve the IEEE118 data case [2] . In this case 
study, the execution was stopped when the objective value was within $1 \%$ of the optimal solution.

Fig.2-5 were the screen shots about CVC from the analysis software based on CPLEX 12.6. It is obvious that model $\mathrm{C}$ has the most similar form of single incidence matrix just as the Fig.1.The red rectangle part of the models in Fig.2-5 represented the correlations between the minimum on/off time constraints and some relative binary variables. The variable $u$ is the only variable in model A. And u and yz for model B. Model C is the same as model B. Model D are just yz. The proper form should have both the elementary binary variables and their differences, so model $\mathrm{D}$ is not the best. Although model B and C both have the best form, there is the summation process in model B, as the dark color blocks in Fig.3. Consequently model C is the best one in CVC from the matrix form. While model A and D are the worst formulation.

The calculation time and peak memory are compared in Fig.6. And the results apparently shows the model C is the optimal model, because it needs the less calculation time, and the less space memory.
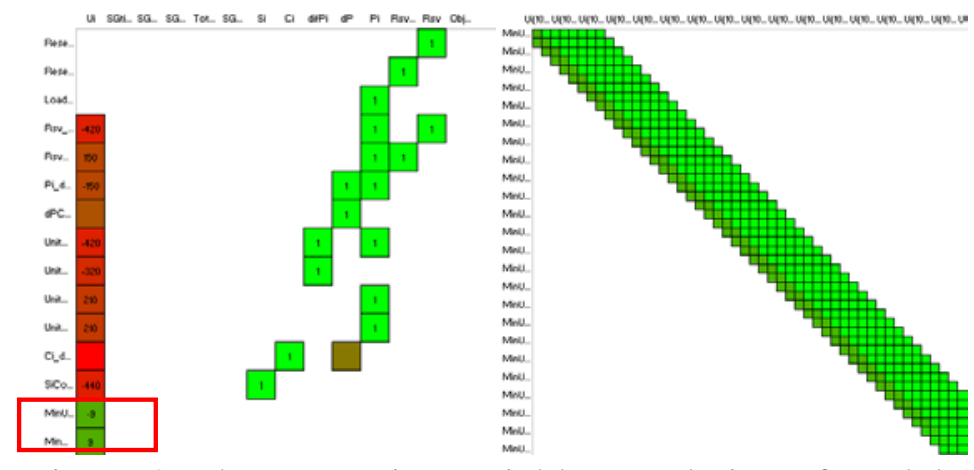

Figure 2. The constraint-variable correlation of model A
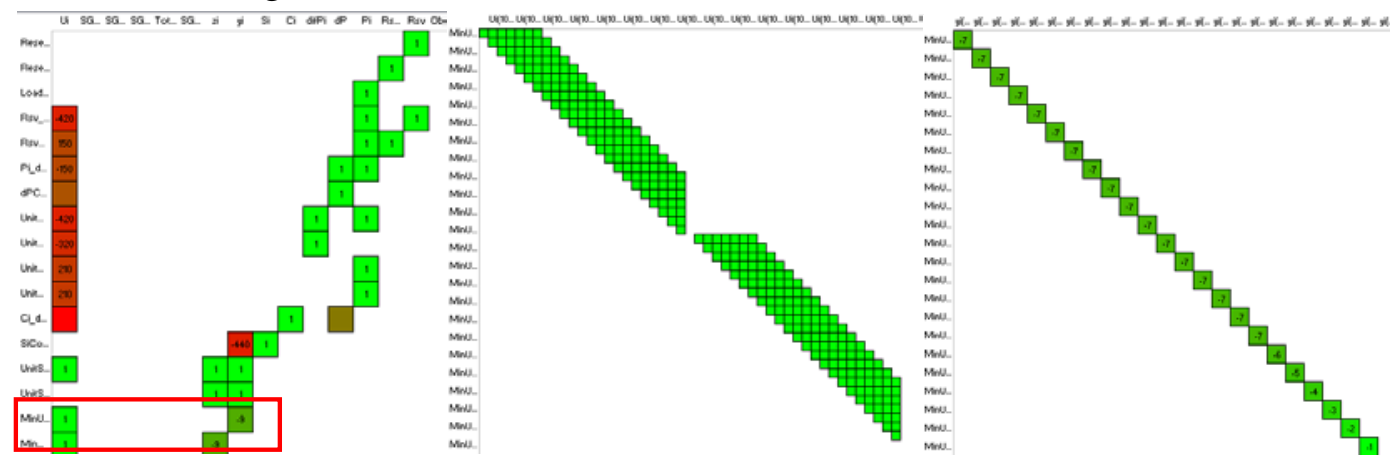

Figure 3. The constraint-variable correlation of model $\mathrm{B}(\mathrm{u}$ on the middle, $\mathrm{z}$ on the right)
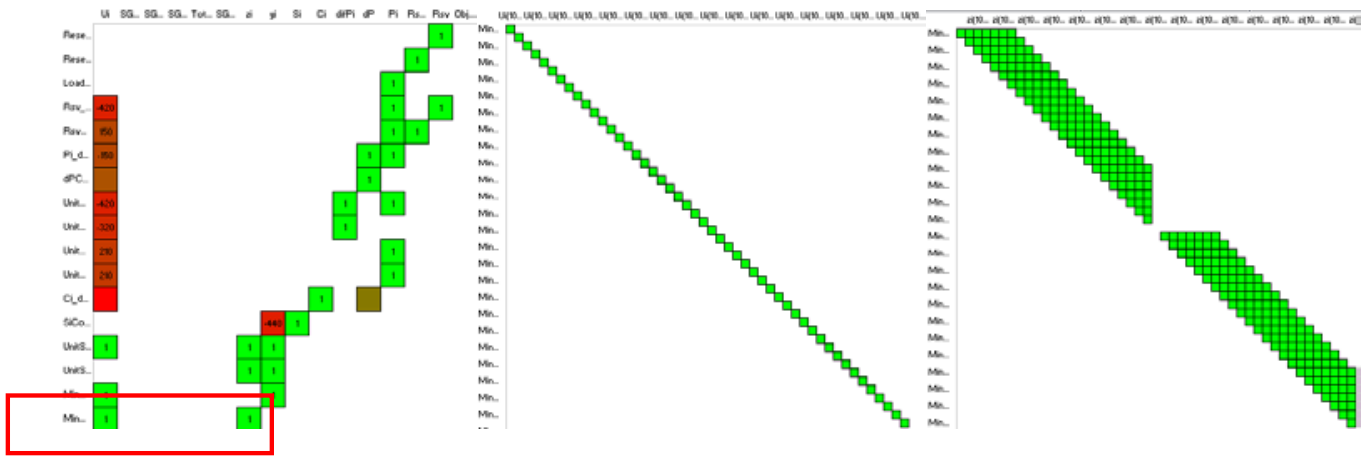

Figure 4. The constraint-variable correlation of model C ( $\mathrm{u}$ on the middle, $\mathrm{z}$ on the right) 

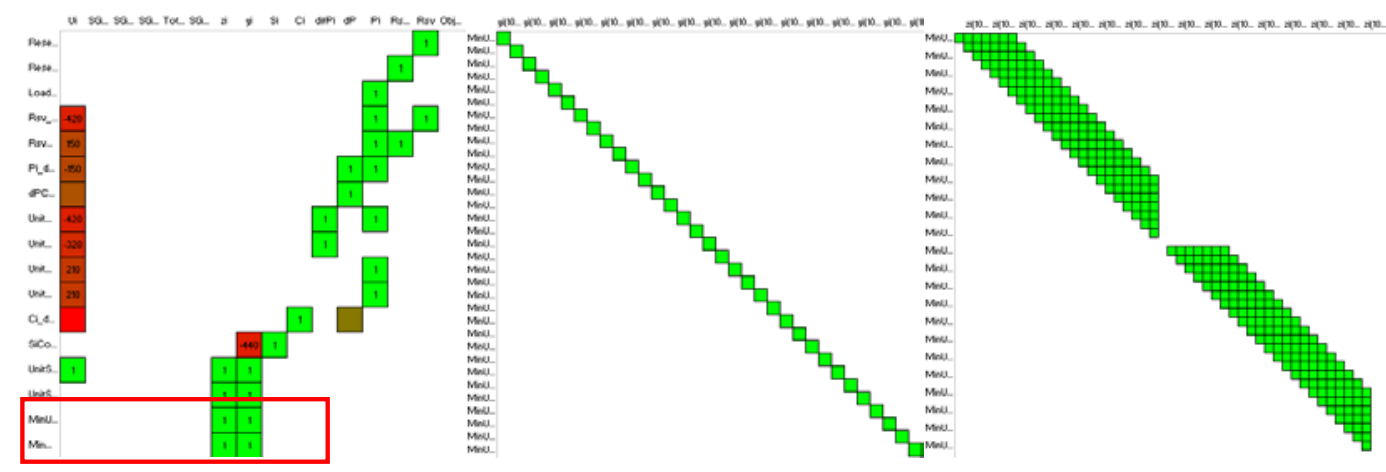

Figure 5. The constraint-variable correlation of model D ( $\mathrm{y}$ on the middle, $\mathrm{z}$ on the right)
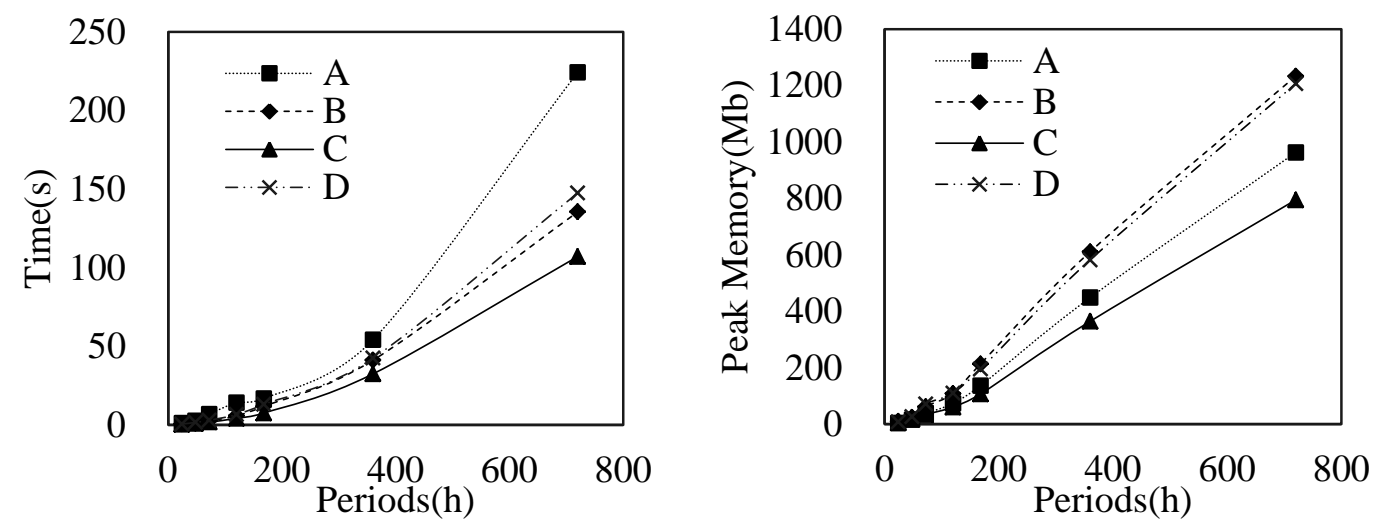

Figure 6. The results of different models

\section{Summary}

The paper put forward CVC criteria to evaluate the models' quality of minimum on/off time constraints. In the condition of different constrained variables, the authors established 4 kinds of models. And they analyzed the CVC of each model mathematically, to select the optimal one (C). At last, numerical results have revealed the best performance of model $\mathrm{C}$ in CVC. The CVC criteria can spread to other areas of models involving correlation. And also the degree of coupling still needs further research, such as the matrix analysis of single correlation.

\section{References}

[1].PADHY N P. Unit Commitment: a Bibliographical Survey[J] . IEEE Transactions on Power Systems, 2004,19(2): 1196-1205.

[2].Li Tao, Shahidehpour M. Price-based Unit Commitment: a Case of Lagrange Relaxation versus Mixed Integer Programming [J]. IEEE Trans on Power Systems, 2005,20(4):2015-2025.

[3].Arroyo J M. , Conejo A J. Optimal Response of a Thermal Unit to an Electricity Spot Market[J]. IEEE Trans on Power System, 2000, 15(3): 1098-1104.

[4].Carrión M, Arroyo J M.., A Computationally Efficient Mixed-integer Linear Formulation for the Thermal Unit Commitment Problem [J]. IEEE Trans on Power Systems, 2006, 21(3):1371-1378.

[5].Ostrowski J., Anjos M.F., Vannelli A., Tight Mixed Integer Linear Programming Formulations for the Unit Commitment Problem [J]. IEEE Trans on Power Systems, 2012, 27(1): 39-46.

[6]. Li Wenyuan, Power System Safe and Economic Operation: Models and Methods [M]. Chongqing University Press, 1988: 222-232. 